\title{
Andamento normale della ionosfera a Roma
}

(Normal behaviour of the ionosphere at Rome)

\author{
P. Domisici (*)
}

Ricevuto il 29 Juglio 1972

Riassunto. - Vengono presentate formule, curve e tabelle per la previsione dell'andamento normale delle principali caratteristiche ionosferiche nella zona di Roma, dedotte dai dati del periodo 1948-1970 dell'Osservatorio ionosferico di Roma dell'Istituto Nazionale di Geofisica.

SUMrary. - Formulae, diagrams and tables obtained through ionospheric data of the Observatory of Rome (Istituto Nazionale di Geofisica) to foresee the normal behaviour of principal ionospheric characteristics at Rome from 1948 to 1970 , are here related.

\section{Premessil}

Radiosondaggi ionosferici verticali sono stati effettuati presso l'Istituto Nazionale di Geofisica a partire dal $1938(1,5.6)$ e ripresi nel 1948, prima nella sede centrale dell'ING, poi nell'Osservatorio ionosferico di Roma-S. Alessio; sono state usate dapprima ionosonde automatiche costruite dall'ING $(1 \div 14 \mathrm{MHz}$ in $40 \mathrm{sec} ; 10 \mathrm{~kW})$, poi, dal 1957, una ionosonda Union Radio MK II $(1,4 \div 15,5 \mathrm{MHz}$ in $180 \mathrm{sec} ; 1 \mathrm{~kW}$ ). I risultati dei sondaggi nel periodo 1948-53 sono stati pubblicati in due fascicoli $(2,4)$; successivamente, sono stati diffusi sotto forma di bollettini mensili.

Vengono qui riportati, con breve commento, i risultati di un'analisi statistica effettuata sui valori mediani mensili delle caratteristiche

(*) Istituto Nazionale di Geofisica, Roma. 
ionosferiche standard del periodo $1948-70$ allo scopo di definire l'andamento normale, cioè merliano non perturbato, della ionosfera a Roma, particolarmente per la previsione lei parametri operativi di radiocollegamenti con riflessione ionosferica circa sulla verticale di Roma; tale analisi integra una precerlente, condotta sui dati del periodo 1948-53 (3).

Assumendo, com'è usuale, il numero $R$ delle macchie solari a indice dell'attività ionizzante del Sole, l'inter'vallo di tempo considerato comprende parte del ciclo 18 (massimo nel 1947, con $R \cong 160$ ), il ciclo 19 (massimo nel 1958, con $R \cong 200$ ) e parte del ciclo 20 (massimo nel 1969 , con $R \simeq 110$ ) dell'attività solare.

Le coordinate dell'Osservatorio ionosferico di Roma-S. Alessio sono le seguenti:

$\begin{array}{lll}\text { latitudine } & \text { geografica } 41,9^{\circ} \mathrm{N} & \text { magnetica } 42,5^{\circ} \mathrm{N}, \\ \text { longitudine } & \text { geografica } 12,5^{\circ} \mathrm{H} & \text { magnetica } 91,9^{\circ}\end{array}$

l'inclinazione magnetica ì $57,1^{\circ}$; la girofrequenza elettronica normale è $1,15 \mathrm{MHz}$ a $200 \mathrm{~km}$ di quota.

Il tempo degli ionogrammi è il tempo del fuso orario $15^{\circ} \mathrm{E}$ $(\mathrm{UT}+1 \mathrm{~h})$. Tal 1957 sono stati effettuati sondaggi ai minuti 00 e 30 di ogni ora; precerlentemente, i sondaggi erano effettuati al minuto 00 di ogni ora.

\section{StRato F'2}

Come strato F'2 s'intende qui la regione $F$ notturna e il più alto (e più ionizzato) dei due strati in cui la regione $\mathrm{F}$ può apparire divisa nelle ore diurne.

Nel periodo considerato la frequenza critica ordinaria, foF', ha variato tra circa 2,0 e circa $15 \mathrm{MHz}$ (densità elettroniche massime corrispondenti: circa $5 \cdot 10^{10}$ e circa $\left.2,8 \cdot 10^{12} \mathrm{~m}^{-3}\right)$.

Sulla base dell'abituale assunzione di una legge di dipendenza lineare, in termini di valori mediani mensili, tra foF2 e il numero $R$ delle macchie solari,

$$
\mathrm{foF}_{n}=a_{n}+b_{h} 10 \div 2
$$

sono stati determinati gli andamenti normali diurni, mese per mese, di foF' per $R=0$ e $R=100$, da cui si sono dedotti i coefficienti orari 
Tabella I - COEPFicINATI ORARI $a_{h}$ (PRIMA RIGA) E $b_{h}$ (SECONDA RIGA) DELLA FORMULA [1].

\begin{tabular}{|c|c|c|c|c|c|c|c|c|c|c|c|c|}
\hline & Gen & Feb & Mar & Apr & Mag & Giu & Lugr & Ago & Set & ott & Nov & Dic \\
\hline 00 & $\begin{array}{l}2,9 \\
1,1\end{array}$ & $\begin{array}{l}2,9 \\
1,7\end{array}$ & $\begin{array}{l}3,0 \\
2,5\end{array}$ & $\begin{array}{l}3,7 \\
2,4\end{array}$ & $\begin{array}{l}3,8 \\
3,0\end{array}$ & $\begin{array}{l}4,4 \\
2,6\end{array}$ & $\begin{array}{l}4,4 \\
2,4\end{array}$ & $\begin{array}{l}3,5 \\
2,2\end{array}$ & $\begin{array}{l}3,1 \\
1,8\end{array}$ & $\begin{array}{l}3,1 \\
1,9\end{array}$ & $\begin{array}{l}3,0 \\
1,7\end{array}$ & $\begin{array}{l}3,2 \\
0,7\end{array}$ \\
\hline 01 & $\begin{array}{l}2,9 \\
1,2\end{array}$ & $\begin{array}{l}2,9 \\
1,7\end{array}$ & $\begin{array}{l}3,0 \\
2,4\end{array}$ & $\begin{array}{l}3,5 \\
2,6\end{array}$ & $\begin{array}{l}3,8 \\
2,7\end{array}$ & $\begin{array}{l}4,0 \\
2,8\end{array}$ & $\begin{array}{l}4,1 \\
2,4\end{array}$ & $\begin{array}{l}3,5 \\
2,3\end{array}$ & $\begin{array}{l}3,2 \\
1,8\end{array}$ & $\begin{array}{l}3,1 \\
1,9\end{array}$ & $\begin{array}{l}3,0 \\
1,8\end{array}$ & $\begin{array}{l}3,1 \\
0,7\end{array}$ \\
\hline 02 & $\begin{array}{l}3,0 \\
1,0\end{array}$ & $\begin{array}{l}2,9 \\
1,6\end{array}$ & $\begin{array}{l}3,0 \\
2,3\end{array}$ & $\begin{array}{l}3,4 \\
2,7\end{array}$ & $\begin{array}{l}3,6 \\
2,7\end{array}$ & $\begin{array}{l}3,8 \\
2,7\end{array}$ & $\begin{array}{l}3,6 \\
2,5\end{array}$ & $\begin{array}{l}3,4 \\
2,2\end{array}$ & $\begin{array}{l}3,2 \\
1,8\end{array}$ & $\begin{array}{l}3,1 \\
1,8\end{array}$ & $\begin{array}{l}3,0 \\
1,7\end{array}$ & $\begin{array}{l}3,1 \\
0,7\end{array}$ \\
\hline 03 & $\begin{array}{l}3,1 \\
0,9\end{array}$ & $\begin{array}{l}3,0 \\
1,4\end{array}$ & $\begin{array}{l}2,9 \\
2,2\end{array}$ & $\begin{array}{l}3,3 \\
2,6\end{array}$ & $\begin{array}{l}3,2 \\
2,6\end{array}$ & $\begin{array}{l}3,6 \\
2,5\end{array}$ & $\begin{array}{l}3,4 \\
2,4\end{array}$ & $\begin{array}{l}3,3 \\
2,1\end{array}$ & $\begin{array}{l}3,1 \\
1,7\end{array}$ & $\begin{array}{l}3,1 \\
1,7\end{array}$ & $\begin{array}{l}3,1 \\
1,5\end{array}$ & $\begin{array}{l}3,0 \\
0,7\end{array}$ \\
\hline 04 & $\begin{array}{l}3,0 \\
0,8\end{array}$ & $\begin{array}{l}3,0 \\
1,4\end{array}$ & $\begin{array}{l}2,8 \\
2,1\end{array}$ & $\begin{array}{l}3,0 \\
2,4\end{array}$ & $\begin{array}{l}3,1 \\
2,6\end{array}$ & $\begin{array}{l}3,6 \\
2,3\end{array}$ & $\begin{array}{l}3,2 \\
2,2\end{array}$ & $\begin{array}{l}3,1 \\
2,0\end{array}$ & $\begin{array}{l}2,9 \\
1,6\end{array}$ & $\begin{array}{l}3,1 \\
1,5\end{array}$ & $\begin{array}{l}3,2 \\
1,2\end{array}$ & $\begin{array}{l}3,0 \\
0,6\end{array}$ \\
\hline 05 & $\begin{array}{l}2,8 \\
0,9\end{array}$ & $\begin{array}{l}2,8 \\
1,5\end{array}$ & $\begin{array}{l}2,6 \\
2,0\end{array}$ & $\begin{array}{l}3,0 \\
2,4\end{array}$ & $\begin{array}{l}4,2 \\
2,2\end{array}$ & $\begin{array}{l}3,9 \\
2,2\end{array}$ & $\begin{array}{l}3,4 \\
2,1\end{array}$ & $\begin{array}{l}3,1 \\
1,9\end{array}$ & $\begin{array}{l}2,5 \\
1,5\end{array}$ & $\begin{array}{l}3,0 \\
1,5\end{array}$ & $\begin{array}{l}3,2 \\
1,0\end{array}$ & $\begin{array}{l}2,9 \\
0,6\end{array}$ \\
\hline 06 & $\begin{array}{l}2,4 \\
1,1\end{array}$ & $\begin{array}{l}2,7 \\
0,9\end{array}$ & $\begin{array}{l}2,9 \\
1,9\end{array}$ & $\begin{array}{l}3,5 \\
2,5\end{array}$ & $\begin{array}{l}4,8 \\
2,2\end{array}$ & $\begin{array}{l}4,6 \\
2,4\end{array}$ & $\begin{array}{l}4,3 \\
2,1\end{array}$ & $\begin{array}{l}4,1 \\
2,0\end{array}$ & $\begin{array}{l}3,3 \\
1,7\end{array}$ & $\begin{array}{l}3,0 \\
1,5\end{array}$ & $\begin{array}{l}2,7 \\
1,3\end{array}$ & $\begin{array}{l}2,5 \\
1,0\end{array}$ \\
\hline 07 & $\begin{array}{l}2,6 \\
1,2\end{array}$ & $\begin{array}{l}3,4 \\
1,6\end{array}$ & $\begin{array}{l}4,5 \\
2,2\end{array}$ & $\begin{array}{l}4,8 \\
2,2\end{array}$ & $\begin{array}{l}5,2 \\
2,2\end{array}$ & $\begin{array}{l}5,3 \\
2,2\end{array}$ & $\begin{array}{l}5,0 \\
2,0\end{array}$ & $\begin{array}{l}4,9 \\
1,9\end{array}$ & $\begin{array}{l}4,3 \\
1,7\end{array}$ & $\begin{array}{l}5,1 \\
1,7\end{array}$ & $\begin{array}{l}3,8 \\
2,4\end{array}$ & $\begin{array}{l}2,8 \\
1,2\end{array}$ \\
\hline 08 & $\begin{array}{l}4,0 \\
2,8\end{array}$ & $\begin{array}{l}4,7 \\
3,3\end{array}$ & $\begin{array}{l}5,0 \\
3,0\end{array}$ & $\begin{array}{l}5,1 \\
2,6\end{array}$ & $\begin{array}{l}5,5 \\
2,1\end{array}$ & $\begin{array}{l}5,7 \\
2,1\end{array}$ & $\begin{array}{l}5,2 \\
2,0\end{array}$ & $\begin{array}{l}5,3 \\
1,9\end{array}$ & $\begin{array}{l}4,9 \\
2,1\end{array}$ & $\begin{array}{l}5,8 \\
2,4\end{array}$ & $\begin{array}{l}5,5 \\
2,8\end{array}$ & $\begin{array}{l}4,5 \\
2,3\end{array}$ \\
\hline 09 & $\begin{array}{r}4,5 \\
4,3\end{array}$ & $\begin{array}{l}5,0 \\
4,9\end{array}$ & $\begin{array}{l}5,5 \\
3,5\end{array}$ & $\begin{array}{l}5,5 \\
3,1\end{array}$ & $\begin{array}{l}5,6 \\
2,3\end{array}$ & $\begin{array}{l}5,7 \\
2,2\end{array}$ & $\begin{array}{l}5,4 \\
2,0\end{array}$ & $\begin{array}{l}5,5 \\
1,9\end{array}$ & $\begin{array}{l}5,1 \\
2,7\end{array}$ & $\begin{array}{l}6,3 \\
3,1\end{array}$ & $\begin{array}{l}6,0 \\
3,5\end{array}$ & $\begin{array}{l}5,1 \\
3,2\end{array}$ \\
\hline 10 & $\begin{array}{l}4,9 \\
4,4\end{array}$ & $\begin{array}{l}5,2 \\
5,1\end{array}$ & $\begin{array}{l}5,8 \\
3,8\end{array}$ & $\begin{array}{l}5,6 \\
3,4\end{array}$ & $\begin{array}{l}5,6 \\
2,5\end{array}$ & $\begin{array}{l}5,6 \\
2,4\end{array}$ & $\begin{array}{l}5,4 \\
2,1\end{array}$ & $\begin{array}{l}5,6 \\
2,0\end{array}$ & $\begin{array}{l}5,2 \\
2,9\end{array}$ & $\begin{array}{l}6,6 \\
3,4\end{array}$ & $\begin{array}{l}6,5 \\
4,3\end{array}$ & $\begin{array}{l}5,8 \\
3,5\end{array}$ \\
\hline 11 & $\begin{array}{l}5,6 \\
4,1\end{array}$ & $\begin{array}{l}5,4 \\
5,2\end{array}$ & $\begin{array}{l}6,1 \\
4,0\end{array}$ & $\begin{array}{l}5,8 \\
3,7\end{array}$ & $\begin{array}{l}5,6 \\
2,7\end{array}$ & $\begin{array}{l}5,4 \\
2,7\end{array}$ & $\begin{array}{l}5,4 \\
2,2\end{array}$ & $\begin{array}{l}5,4 \\
2,4\end{array}$ & $\begin{array}{l}5,4 \\
2,9\end{array}$ & $\begin{array}{l}6,8 \\
3,7\end{array}$ & $\begin{array}{l}6,8 \\
3,9\end{array}$ & $\begin{array}{l}6,0 \\
4,2\end{array}$ \\
\hline 12 & $\begin{array}{l}6,0 \\
3,7\end{array}$ & $\begin{array}{l}5,6 \\
5,1\end{array}$ & $\begin{array}{l}6,3 \\
4,1\end{array}$ & $\begin{array}{l}5,4 \\
3,9\end{array}$ & $\begin{array}{l}5,6 \\
2,9\end{array}$ & $\begin{array}{l}5,3 \\
2,8\end{array}$ & $\begin{array}{l}5,4 \\
2,6\end{array}$ & $\begin{array}{l}5,2 \\
2,8\end{array}$ & $\begin{array}{l}5,5 \\
3,0\end{array}$ & $\begin{array}{l}6,9 \\
3,8\end{array}$ & $\begin{array}{l}7,0 \\
3,6\end{array}$ & $\begin{array}{l}5,4 \\
4,5\end{array}$ \\
\hline 13 & $\begin{array}{l}6,2 \\
3,3\end{array}$ & $\begin{array}{l}5,6 \\
5,0\end{array}$ & $\begin{array}{l}6,1 \\
4,1\end{array}$ & $\begin{array}{l}5,9 \\
4,0\end{array}$ & $\begin{array}{l}5,5 \\
3,1\end{array}$ & $\begin{array}{l}5,3 \\
2,8\end{array}$ & $\begin{array}{l}5,5 \\
2,3\end{array}$ & $\begin{array}{l}5,3 \\
2,7\end{array}$ & $\begin{array}{l}5,6 \\
2,9\end{array}$ & $\begin{array}{l}6,9 \\
3,7\end{array}$ & $\begin{array}{l}6,6 \\
3,8\end{array}$ & $\begin{array}{l}5,2 \\
4,3\end{array}$ \\
\hline 14 & $\begin{array}{l}5,9 \\
3,4\end{array}$ & $\begin{array}{l}5,5 \\
4,9\end{array}$ & $\begin{array}{l}6,0 \\
4,2\end{array}$ & $\begin{array}{l}6,0 \\
3,8\end{array}$ & $\begin{array}{l}\overline{5}, 5 \\
3,1\end{array}$ & $\begin{array}{l}5,3 \\
2,7\end{array}$ & $\begin{array}{l}5,3 \\
2,3\end{array}$ & $\begin{array}{l}5,4 \\
2,5\end{array}$ & $\begin{array}{l}5,6 \\
2,9\end{array}$ & $\begin{array}{l}6,8 \\
3,6\end{array}$ & $\begin{array}{l}6,2 \\
4,1\end{array}$ & $\begin{array}{l}5,1 \\
4,2\end{array}$ \\
\hline 15 & $\begin{array}{l}5,3 \\
3,7\end{array}$ & $\begin{array}{l}5,3 \\
4,8\end{array}$ & $\begin{array}{l}5,8 \\
4,2\end{array}$ & $\begin{array}{l}6,0 \\
3,5\end{array}$ & $\begin{array}{l}5,6 \\
3,0\end{array}$ & $\begin{array}{l}5,3 \\
2,6\end{array}$ & $\begin{array}{l}5,3 \\
2,2\end{array}$ & $\begin{array}{l}5,1 \\
2,5\end{array}$ & $\begin{array}{l}5,6 \\
2,8\end{array}$ & $\begin{array}{l}6,7 \\
3,5\end{array}$ & $\begin{array}{l}5,9 \\
4,2\end{array}$ & $\begin{array}{l}5,0 \\
3,9\end{array}$ \\
\hline 16 & $\begin{array}{l}4,6 \\
3,8\end{array}$ & $\begin{array}{l}5,0 \\
4,6\end{array}$ & $\begin{array}{l}5,7 \\
4,1\end{array}$ & $\begin{array}{l}5,9 \\
3,4\end{array}$ & $\begin{array}{l}5,8 \\
2,8\end{array}$ & $\begin{array}{l}5,4 \\
2,1\end{array}$ & $\begin{array}{l}5,3 \\
2,2\end{array}$ & $\begin{array}{l}5,2 \\
2,4\end{array}$ & $\begin{array}{l}5,6 \\
2,7\end{array}$ & $\begin{array}{l}6,6 \\
3,3\end{array}$ & $\begin{array}{l}5,4 \\
3,7\end{array}$ & $\begin{array}{l}4,6 \\
3,5\end{array}$ \\
\hline 17 & $\begin{array}{l}3,7 \\
3,9\end{array}$ & $\begin{array}{l}4,7 \\
4,2\end{array}$ & $\begin{array}{l}5,6 \\
4,1\end{array}$ & $\begin{array}{l}5,7 \\
3,4\end{array}$ & $\begin{array}{l}6,0 \\
2,6\end{array}$ & $\begin{array}{l}5,6 \\
1,9\end{array}$ & $\begin{array}{l}5,3 \\
2,2\end{array}$ & $\begin{array}{l}5,3 \\
2,2\end{array}$ & $\begin{array}{l}5,8 \\
2,4\end{array}$ & $\begin{array}{l}6,4 \\
3,1\end{array}$ & $\begin{array}{l}4,3 \\
3,7\end{array}$ & $\begin{array}{l}3,4 \\
3,8\end{array}$ \\
\hline 18 & $\begin{array}{l}2,6 \\
3,8\end{array}$ & $\begin{array}{l}3,6 \\
4,5\end{array}$ & $\begin{array}{l}5,6 \\
3,7\end{array}$ & $\begin{array}{l}5,8 \\
2,9\end{array}$ & $\begin{array}{l}6,3 \\
2,3\end{array}$ & $\begin{array}{l}5,4 \\
1,8\end{array}$ & $\begin{array}{l}5,5 \\
2,0\end{array}$ & $\begin{array}{l}5,8 \\
2,0\end{array}$ & $\begin{array}{l}6,0 \\
2,0\end{array}$ & $\begin{array}{l}5,4 \\
2,6\end{array}$ & $\begin{array}{l}3,2 \\
3,6\end{array}$ & $\begin{array}{l}2,9 \\
3,1\end{array}$ \\
\hline 19 & $\begin{array}{l}2,8 \\
2,7\end{array}$ & $\begin{array}{l}3,2 \\
3,9\end{array}$ & $\begin{array}{l}4,7 \\
3,3\end{array}$ & $\begin{array}{l}5,4 \\
2,6\end{array}$ & $\begin{array}{l}6,6 \\
1,9\end{array}$ & $\begin{array}{l}6,7 \\
1,6\end{array}$ & $\begin{array}{l}6,0 \\
2,0\end{array}$ & $\begin{array}{l}6,7 \\
1,4\end{array}$ & $\begin{array}{l}6,0 \\
1,8\end{array}$ & $\begin{array}{l}4,5 \\
2,5\end{array}$ & $\begin{array}{l}3,1 \\
2,9\end{array}$ & $\begin{array}{l}3,0 \\
2,0\end{array}$ \\
\hline 20 & $\begin{array}{l}2,9 \\
1,6\end{array}$ & $\begin{array}{l}3,0 \\
3,0\end{array}$ & $\begin{array}{l}3,8 \\
2,8\end{array}$ & $\begin{array}{l}5,8 \\
2,5\end{array}$ & $\begin{array}{l}6,8 \\
1,3\end{array}$ & $\begin{array}{l}7,4 \\
1,0\end{array}$ & $\begin{array}{l}6,5 \\
1,7\end{array}$ & $\begin{array}{l}6,9 \\
1,1\end{array}$ & $\begin{array}{l}5,3 \\
1,8\end{array}$ & $\begin{array}{l}4,0 \\
2,0\end{array}$ & $\begin{array}{l}3,1 \\
2,3\end{array}$ & $\begin{array}{l}3,1 \\
1,1\end{array}$ \\
\hline 21 & $\begin{array}{l}2,6 \\
1,4\end{array}$ & $\begin{array}{l}2,9 \\
2,5\end{array}$ & $\begin{array}{l}3,4 \\
2,9\end{array}$ & $\begin{array}{l}5,5 \\
2,0\end{array}$ & $\begin{array}{l}6,6 \\
1,2\end{array}$ & $\begin{array}{l}6,8 \\
1,3\end{array}$ & $\begin{array}{l}6,3 \\
1,5\end{array}$ & $\begin{array}{l}6,2 \\
1,2\end{array}$ & $\begin{array}{l}4,7 \\
1,6\end{array}$ & $\begin{array}{l}3,3 \\
2,3\end{array}$ & $\begin{array}{l}2,7 \\
2,3\end{array}$ & $\begin{array}{l}3,0 \\
0,8\end{array}$ \\
\hline 22 & $\begin{array}{l}2,6 \\
1,4\end{array}$ & $\begin{array}{l}2,1 \\
1,7\end{array}$ & $\begin{array}{l}3,2 \\
2,8\end{array}$ & $\begin{array}{l}4,0 \\
2,6\end{array}$ & $\begin{array}{l}5,2 \\
2,1\end{array}$ & $\begin{array}{l}5,8 \\
1,8\end{array}$ & $\begin{array}{l}5,4 \\
1,8\end{array}$ & $\begin{array}{l}5,3 \\
1,4\end{array}$ & $\begin{array}{l}4,2 \\
1,6\end{array}$ & $\begin{array}{l}3,2 \\
2,2\end{array}$ & $\begin{array}{l}2,7 \\
1,9\end{array}$ & $\begin{array}{l}2,9 \\
0,8\end{array}$ \\
\hline 23 & $\begin{array}{l}2,8 \\
1,2\end{array}$ & $\begin{array}{l}2,9 \\
1,7\end{array}$ & $\begin{array}{l}3,2 \\
2,4\end{array}$ & $\begin{array}{l}3,7 \\
2,5\end{array}$ & $\begin{array}{l}4,3 \\
2,6\end{array}$ & $\begin{array}{l}5,2 \\
2,1\end{array}$ & $\begin{array}{l}4,7 \\
2,3\end{array}$ & $\begin{array}{l}4,4 \\
1,7\end{array}$ & $\begin{array}{l}3,6 \\
1,7\end{array}$ & $\begin{array}{l}3,1 \\
2,1\end{array}$ & $\begin{array}{l}2,9 \\
1,7\end{array}$ & $\begin{array}{l}3,0 \\
0,7\end{array}$ \\
\hline
\end{tabular}




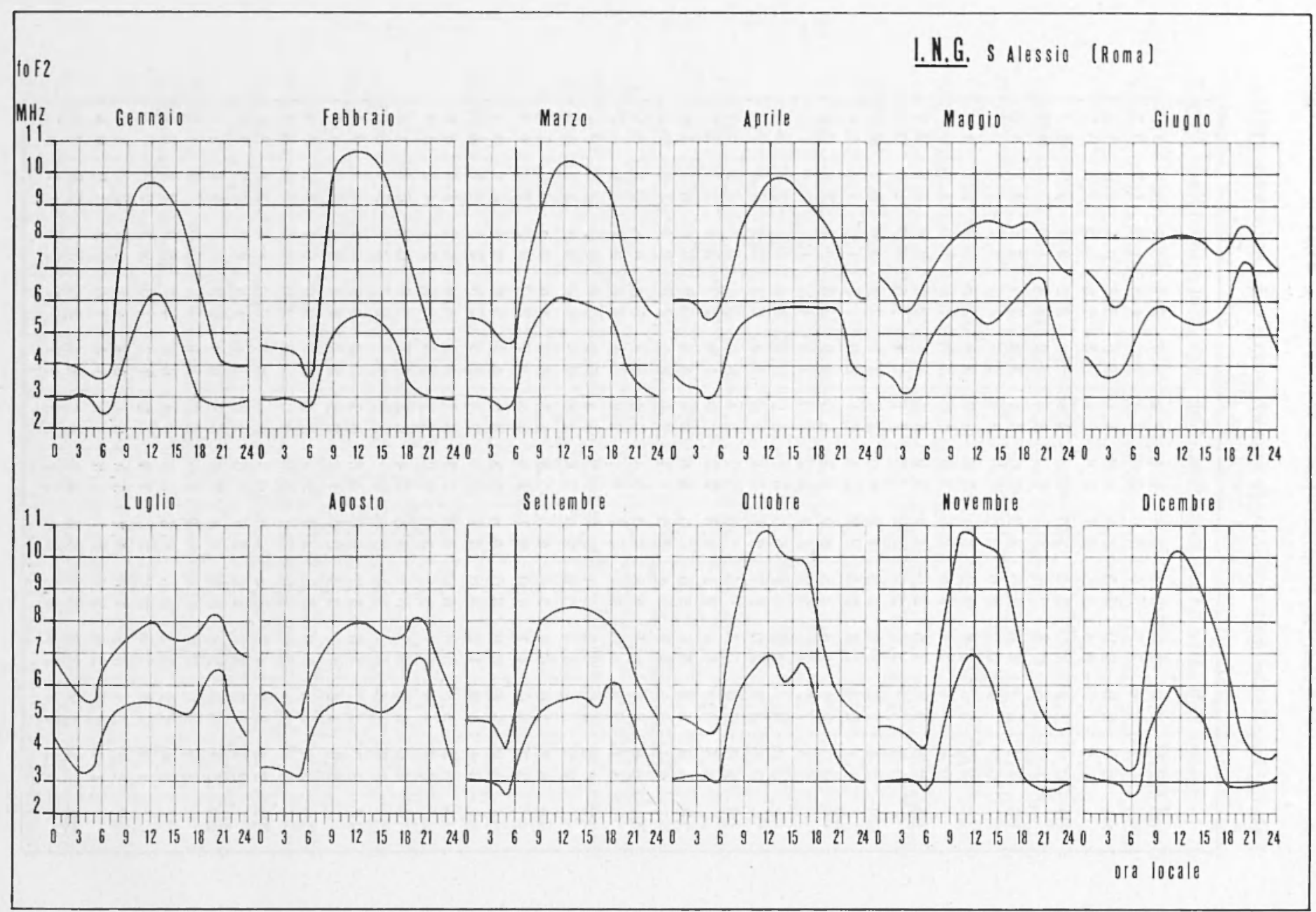

Fig. 1 - Andamenti diurni mensili normali di foF2 per $R=0$ (curve inferiori) e $R=100$ (curve superiori). 
di dipendenza lineare riportati nella Tab. I. Gli anclamenti anzidetti, sottoposti a perequazione grafica, sono riportati nella fig. 1.

Ia [1] costituisce la formula di previsione dell'andamento normale della caratteristica ionosferica foFe sulla verticale di Roma. Sulla precisione dei coefficienti $a_{\mu}, b_{h}$ molto influisce la dispersione dei valori, che risulta, grossolanamente parlando, crescente al crescere di foF2, e quindi rilevante nelle ore meridiane di mesi invernali, specie in periorli di alta attività solare. ì da ritenere peraltro che l'imprecisione con cui sono determinate le rette rappresentate dalla [1] non sia tale da compromettere l'utilita di questa relazione ai fini di una previsione di massima. Piuttosto, seri lubbi possono essere avanzati sulla generale valiclità di una relazione lineare tra foF'2 e $R$ : ci riferiamo al fatto, ben noto, della cosidletta "saturazione" di foF", cioè al fatto che i coefficienti $b_{n}$ risultano essere in realtà funzioni decrescenti di $R$. Converrà, al riguardo, esaminare alcuni risultati del trattamento statistico dei clati.

Cosi, la fig. 2 mostra gli andamenti dei valori merliani mensili di $R$, delle merliane mensili di foF' alle ore 00 (foF $2_{00}$ ) e alle ore 12 $\left(\right.$ foF $2_{12}$ ) e della frequenza critica media cliurna,

$$
\text { foF }_{2}=\frac{1}{24} \sum_{00}^{23} \text { for } 2_{\mu}
$$

ristretti, per comodità di presentazione, al decennio 1960-69. Ad onta della grossa perturbazione costituita dall'andamento stagionale, si nota un sensibile parallelismo tra gli andamenti a lungo termine. Tale parallelismo è ben messo in eviclenza nella fig. 3 , in cui ai valori mediani mensili sono stati sostituiti i valori (sopralineati) ottenuti con una media mobile annua simmetrica a 13 mesi (con il che s'attenua drasticamente l'anclamento stagionale); è riportato anche l'andamento della media mobile annua $Q$, della grandeza

$$
Q=\frac{1}{i \dot{i}} \sum_{00}^{23}(\mathrm{foF} 2)^{2}
$$

costituente un indice medio della densità elettronica massima dello strato. Nella fig. 4 è poi riportato l'andamento di foF $\overline{2}_{12}$ con $R$. Anche se operando su base locale non è possibile trarre alcuna conclusione generale, l'esame di queste figure porta a concludere che per quanto 


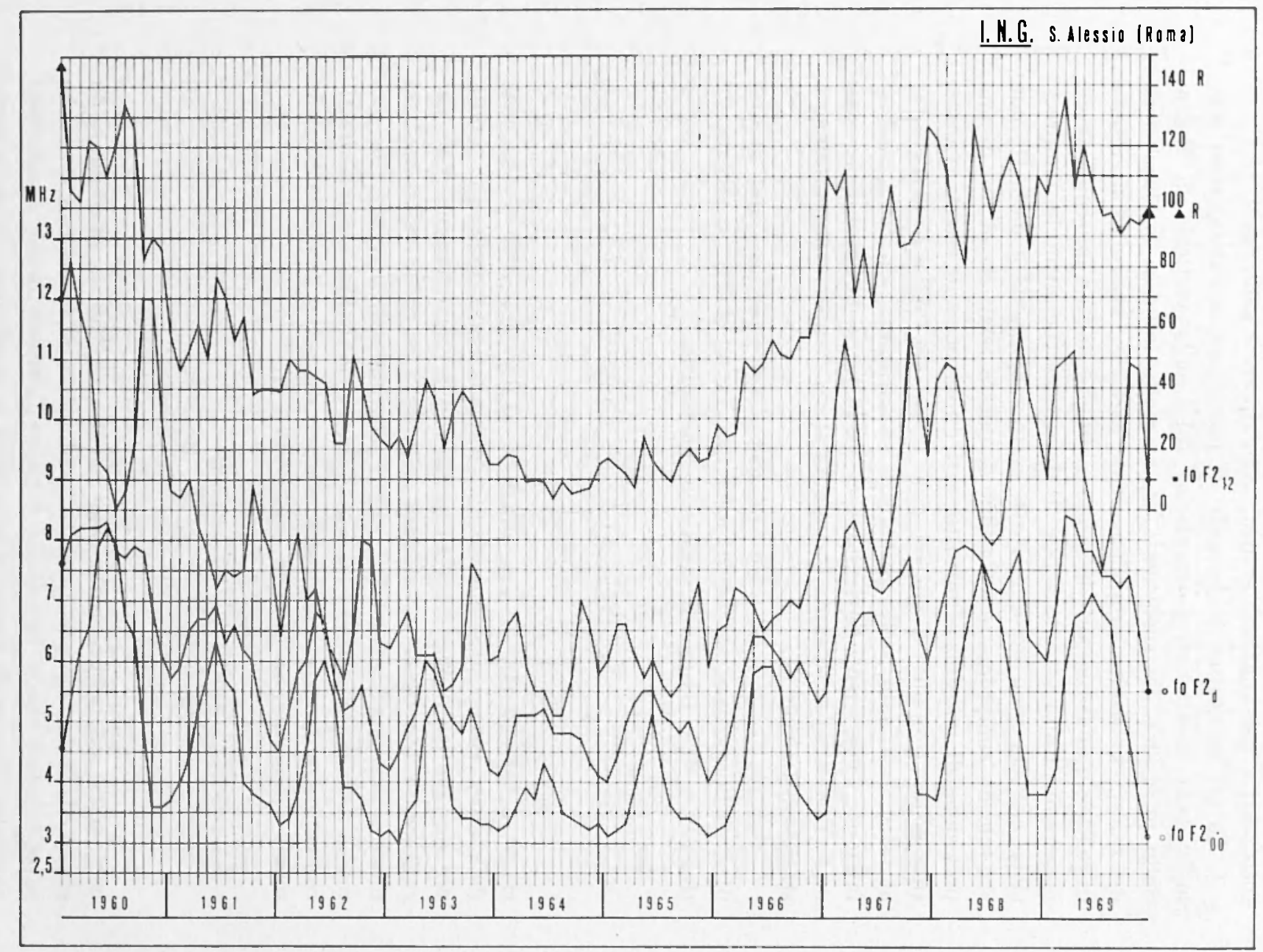

Fir. 2 - Andamento dei valori mediani mensili di $R$, foF $2_{12}$, foF $2_{d}$, foF $2_{0 n}$ nel decennio 1960-1969. 


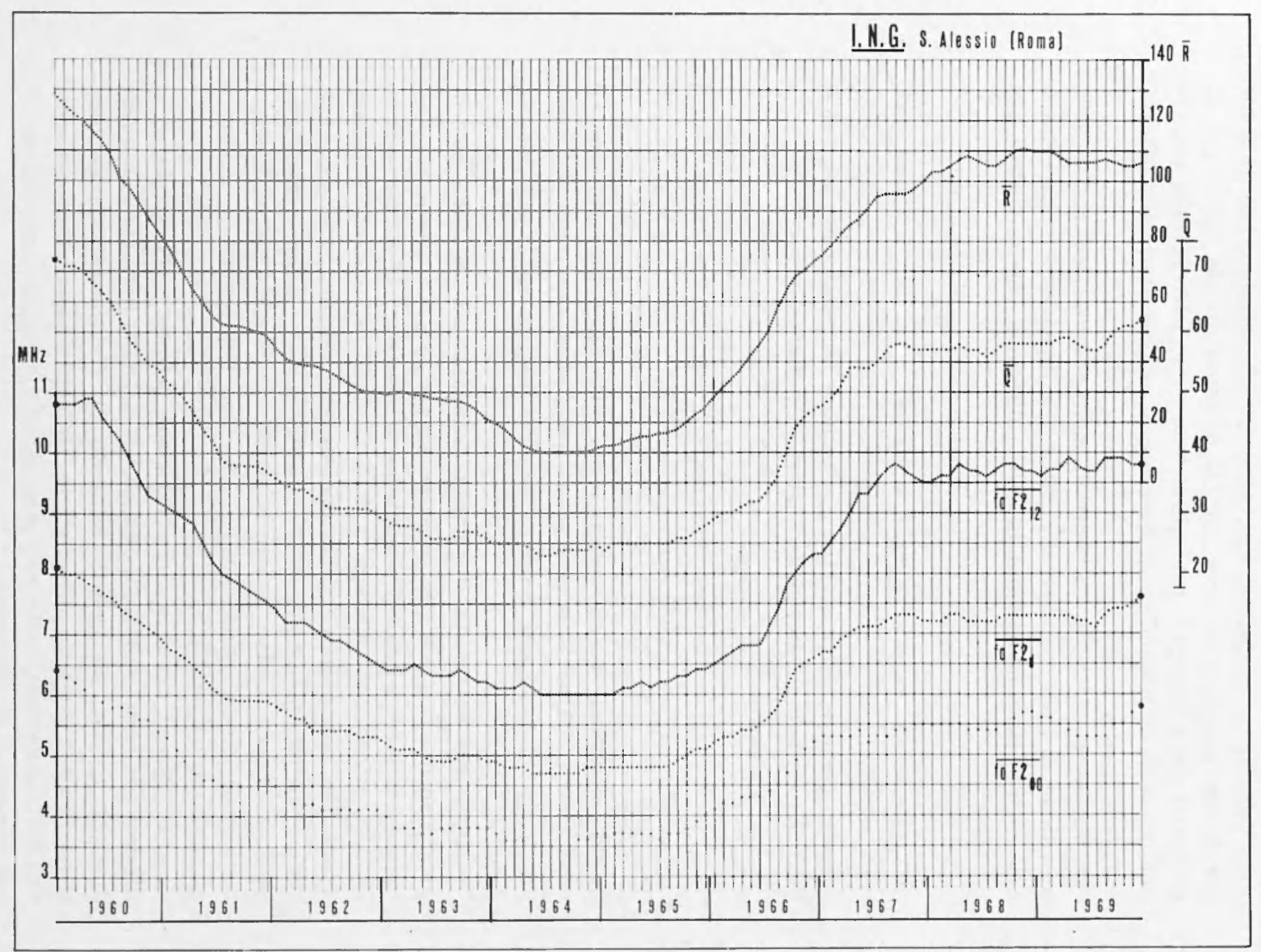

Fig. 3. - Andamento delle medie mobili annue di $R, Q$, foF $2_{12}$, foF $2_{d}$, foF $2_{00}$ nel decennio $1960-1969$. 
riguarda i dati di Roma, la [1] è accettabile per una previsione di massima soltanto in periorli di non alta attivita solare, e precisamente fer $R$ non maggiore di circa 120 ; al di sopra di tale valore per $R$, gli scarti dalla lineariti, per saturazione e per isteresi, sono tali che non si vede la possibilita di ricorrere a rappresentazioni analitiche semplici.

Poiché, com'è noto, da parte di molti si preferisce, ai tini della previsione di foF 2 , mettere a profitto una dipendenza lineare tra $\bar{Q}$ e $R$, i stata fatta un'indagine in questa direzione, che ha portato alla relazione:

$$
(?=18+0,38 K,
$$

soggetta peraltro, come la [1], a una validità limitata a non alti valori di $R$. La formula di previsione è allora la seguente:

$$
\text { foF } 2_{h M}=\text { foF } 2_{h M o}\left(\frac{\bar{Q}_{M p}}{\bar{Q}_{M o}}\right)^{1 / 2},
$$

in cui i deponenti $h$ e $M$ indicano una certa ora e un certo mese, il deponente o indica valori osservati a quell'ora di quel mese rell'anno precerlente, il deponente $p$ indica il valore di $Q$ previsto, cioè dedotto dalla [: 1].

Oltre che su questa singolarità dell'andamento di foF' con $R$, genericamente non lineare, cogliamo l'occasione per richiamare l'attenzione sulle altre ben note singolarità dell'andamento diurno e annuo di foF', evirlenziate nelle figg. $1,2,5$ (in quest'ultima, sono stati utilizzati dati non perequati). Sinteticamente parlando, lo strato "notturno" ha un solo picco, estivo, mentre quello "diurno" ha picchi equinoziali e un minimo estivo; lo strato "invernale " ha un massimo meridliano, mentre quello "estivo" ha una depressione merilliana e un massimo pomerirliano.

Per quanto riguarda l'altezza virtuale minima dello strato, ei si riferisce alla caratteristica ionosferica $h^{\prime} \mathrm{F}$ nelle ore notturne e in quelle diurne in cui non sia presente lo strato $\mathrm{F} 1$, e alla caratteristica ionosferica $h^{\prime} \mathrm{F} 2$ in presenza di tale strato. In merito all'andamento diurno, una netta differenza esiste tra i mesi in cui è osservabile uno strato F1 ben formato e gli altri mesi. 


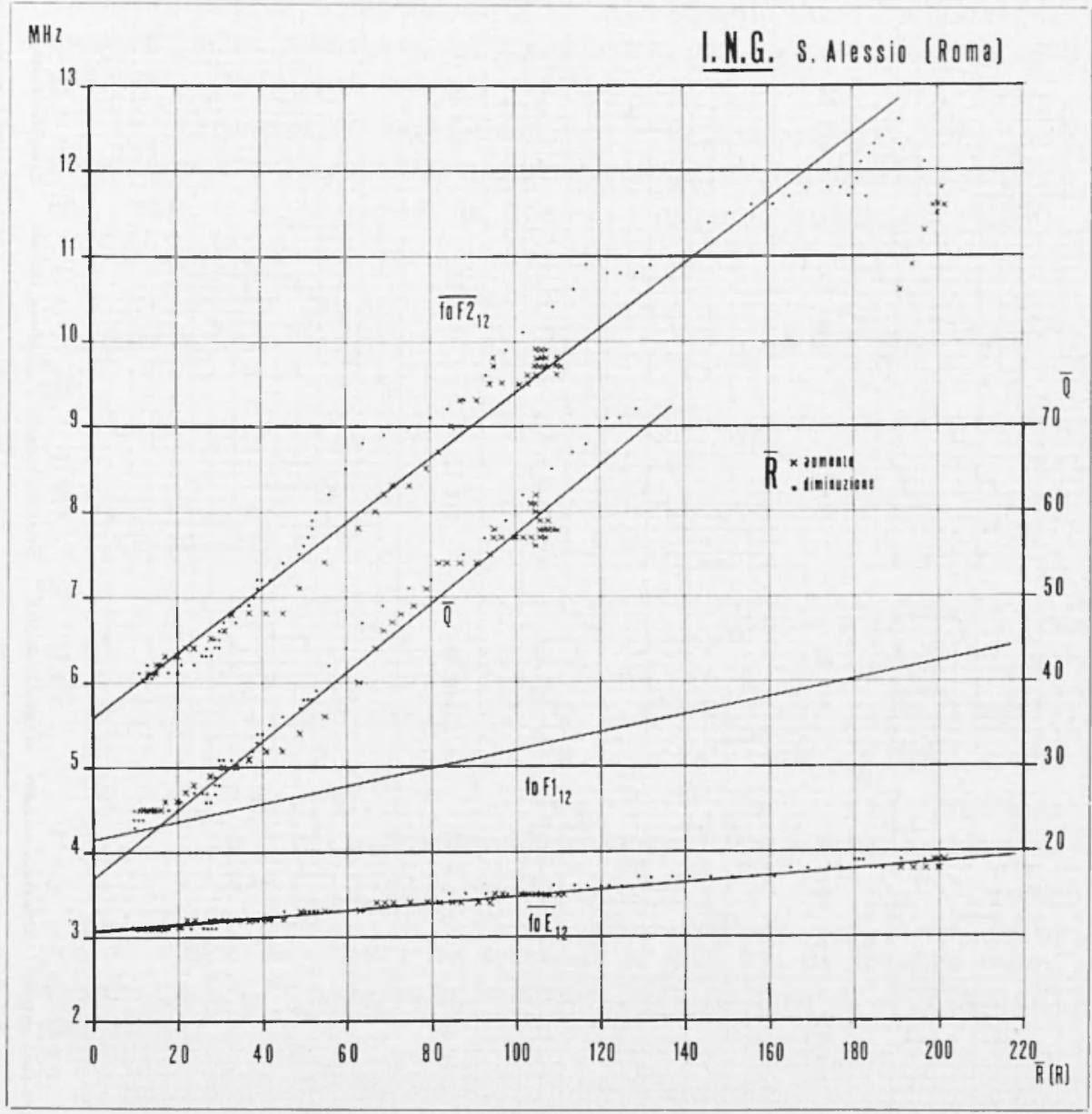

Fig. 4. - Correlogramma di foF $2_{12}$, fo $\mathrm{E}_{12}$, foF $1_{12}$ verso $R$. 


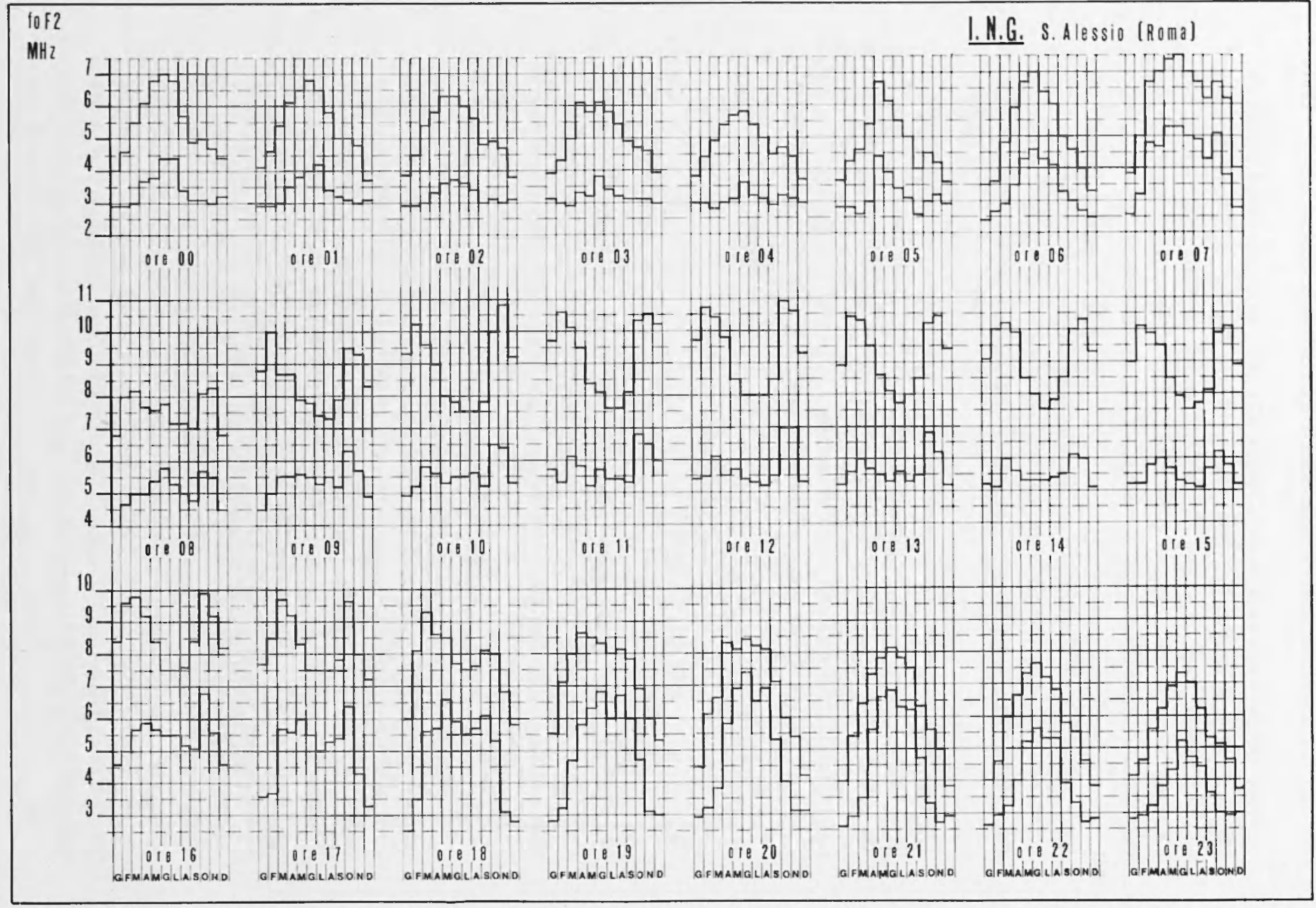

Fig. 5. - Andamenti annui orarî normali di foF2 per $R=0$ (eurve inferiori) ed $R-100$ (curve superiori). 
Nel primo caso, che, statisticamente parlando si verifica a Roma nei mesi a declinazione solare nettamente positiva (Aprile, Maggio, Giugno, Luglio, Agosto), l'andamento medio è quello della curva aba della fig. 6 (la curva e rappresenta l'andamento medio dell'altezza virtuale minima dello strato F1); nel secondo caso, l'andamento medio è quello della curva aca. Non ì risultata una significativa variazione con l'attività solare.

Per le previsioni ionosferiche per le radiocomunicazioni ha importanza anche il fattore di trasmissione obliqua a $3000 \mathrm{~lm}, \mathrm{M}(3000) \mathrm{F} 2$, che, come si sa, determina la frequenza massima utilizzabile (MUF)

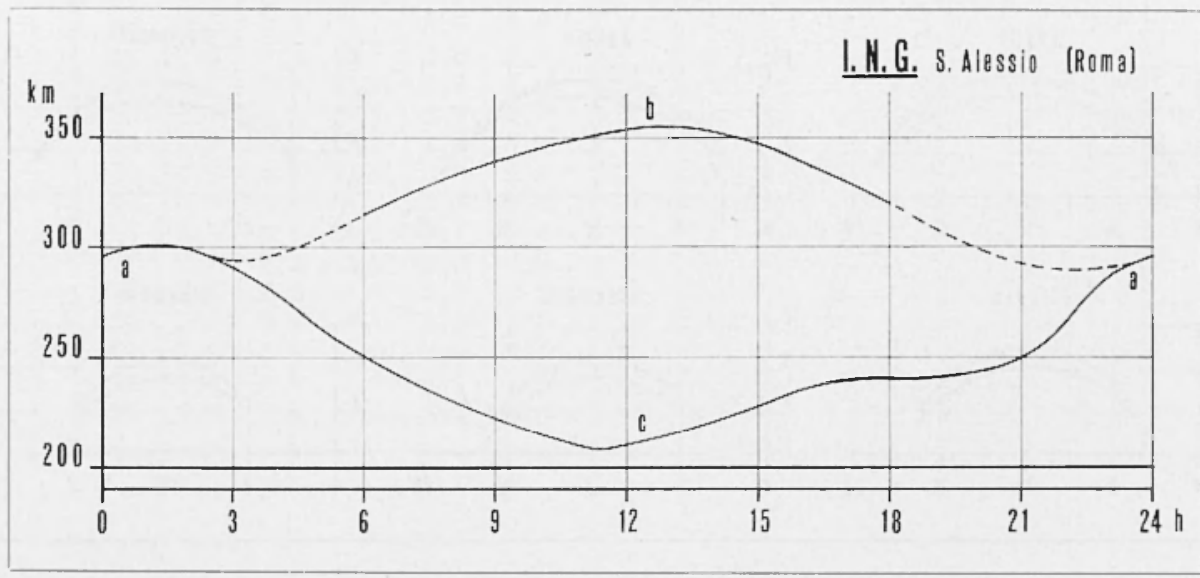

Fig. 6. - Andamento diurno medio di h'F e h'F2.

per un radiocollegamento fra terminali a $3000 \mathrm{~km}$ di distanza ortodromica con riflessione sulla verticale della stazione di rilevamento ionosferico,

$$
\operatorname{MUF}(3000) \mathrm{F}^{2}=\text { foF } 2 \cdot \mathrm{M}(3000) \mathrm{F}^{2},
$$

e successivamente, mediante l'uso di noti nomogrammi, consente la determinazione della MUF per ogni altra distanza sino a circa $4000 \mathrm{~km}$. Come per l'altezza virtuale minima, anche per tale fattore s'è constatata una dipendenza da $R$ piuttosto modesta, non molto maggiore della dispersione che, per varie cause, caratterizza tali dati; cosi, s'è deciso di assumere come dati dell'andamento normale i valori mediani dei valori mediani mensili correnti. 


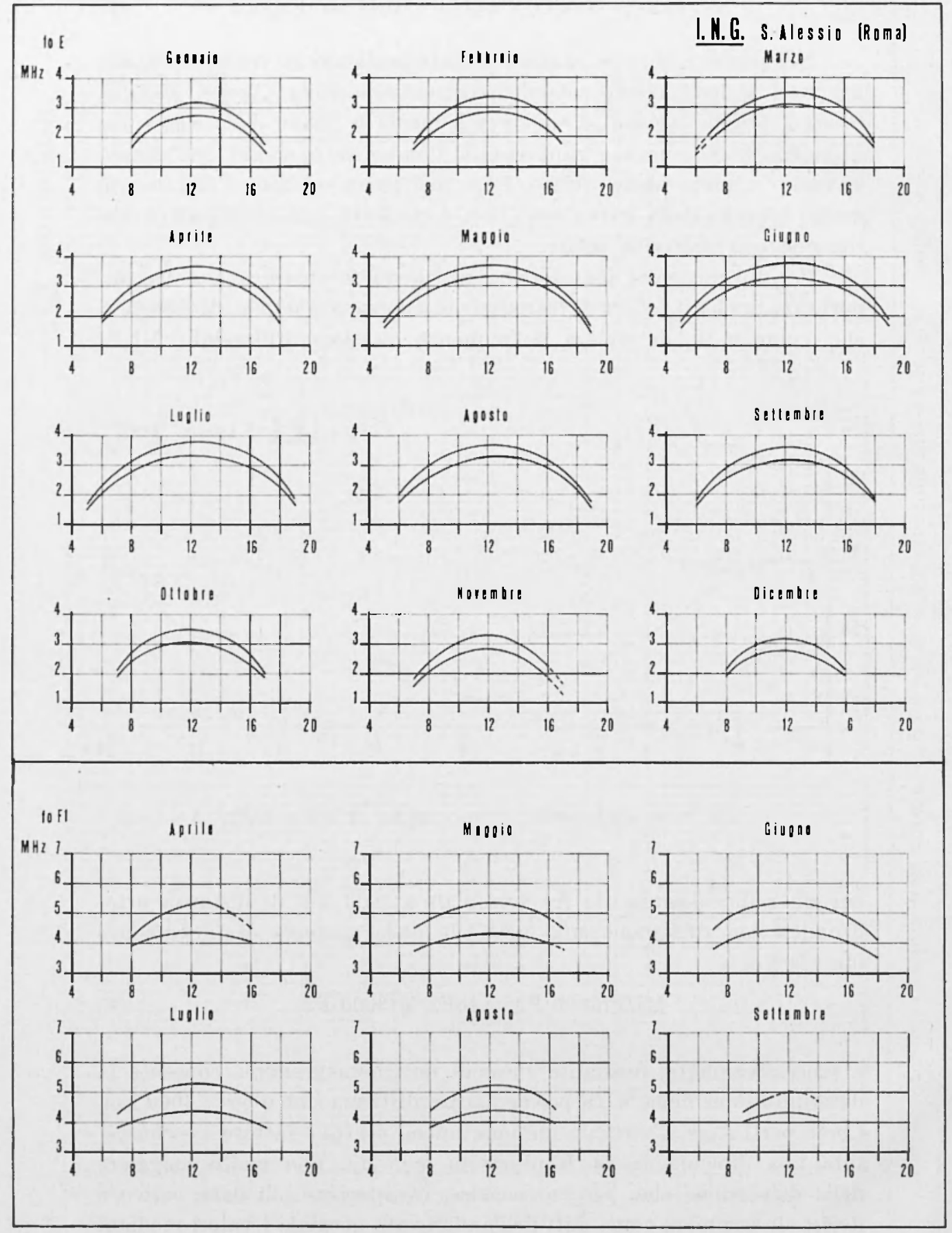

Fig. 7. - Andamenti diurni mensili normali di foFl e foE per $R-0$ (curve inferiori) e $R=100$ (curve superiori). 
Tali dati sono riportati nella seguente Tab. II.

Tabella II - FatToRI ORARI DI TRASMISSIONE M(3000)F2

\begin{tabular}{|c|c|c|c|c|c|c|c|c|c|c|c|c|}
\hline & Gen & Feb & Mar & Apr & Mag & Giu & Lug & Ago & Set & Ott & Nov & Dic \\
\hline 00 & 3,0 & 2,9 & 2,8 & 2,7 & 2,7 & 2,8 & 2,8 & 2,7 & 2,7 & 2,7 & 2,8 & 2,8 \\
01 & 3,0 & 2,9 & 2,8 & 2,7 & 2,7 & 2,7 & 2,7 & 2,7 & 2,7 & 2,7 & 2,8 & 2,8 \\
02 & 2,9 & 2,9 & 2,8 & 2,7 & 2,7 & 2,7 & 2,7 & 2,7 & 2,8 & 2,8 & 2,8 & 2,8 \\
03 & 2,9 & 2,9 & 2,9 & 2,7 & 2,7 & 2,8 & 2,7 & 2,7 & 2,8 & 2,8 & 2,9 & 2,9 \\
04 & 3,0 & 2,9 & 2,9 & 2,8 & 2,8 & 2,8 & 2,8 & 2,8 & 2,9 & 2,8 & 2,9 & 2,9 \\
05 & 3,1 & 3,0 & 3,0 & 2,9 & 2,9 & 2,8 & 2,9 & 3,0 & 2,9 & 3,0 & 3,0 & 3,0 \\
06 & 3,2 & 3,1 & 3,1 & 3,2 & 3,1 & 2,9 & 2,9 & 3,1 & 3,2 & 3,1 & 3,2 & 3,2 \\
07 & 3,3 & 3,3 & 3,4 & 3,2 & 3,1 & 3,0 & 3,0 & 3,1 & 3,2 & 3,2 & 3,3 & 3,3 \\
08 & 3,4 & 3,3 & 3,3 & 3,1 & 3,1 & 3,0 & 3,0 & 3,1 & 3,2 & 3,3 & 3,4 & 3,4 \\
09 & 3,5 & 3,4 & 3,2 & 3,1 & 3,1 & 3,0 & 3,0 & 3,1 & 3,2 & 3,3 & 3,3 & 3,4 \\
10 & 3,5 & 3,4 & 3,1 & 3,0 & 3,0 & 2,9 & 2,9 & 3,1 & 3,2 & 3,2 & 3,3 & 3,4 \\
11 & 3,5 & 3,3 & 3,1 & 2,9 & 2,9 & 2,9 & 2,9 & 3,0 & 3,2 & 3,2 & 3,3 & 3,4 \\
12 & 3,5 & 3,3 & 3,0 & 2,9 & 2,9 & 2,9 & 2,8 & 3,0 & 3,1 & 3,2 & 3,2 & 3,4 \\
13 & 3,4 & 3,3 & 3,1 & 2,9 & 2,9 & 2,9 & 2,8 & 2,9 & 3,1 & 3,1 & 3,2 & 3,3 \\
14 & 3,3 & 3,3 & 3,1 & 3,0 & 3,0 & 2,9 & 2,9 & 2,9 & 3,1 & 3,1 & 3,2 & 3,3 \\
15 & 3,3 & 3,3 & 3,2 & 3,0 & 3,0 & 2,9 & 2,9 & 3,0 & 3,1 & 3,1 & 3,3 & 3,4 \\
16 & 3,4 & 3,4 & 3,2 & 3,1 & 3,0 & 2,9 & 2,9 & 3,0 & 3,1 & 3,1 & 3,3 & 3,4 \\
17 & 3,5 & 3,4 & 3,2 & 3,1 & 3,0 & 2,9 & 2,9 & 3,0 & 3,1 & 3,2 & 3,3 & 3,4 \\
18 & 3,4 & 3,3 & 3,2 & 3,0 & 2,9 & 2,9 & 2,9 & 3,0 & 3,1 & 3,3 & 3,2 & 3,3 \\
19 & 3,2 & 3,2 & 3,2 & 3,1 & 3,0 & 2,9 & 2,9 & 3,0 & 3,1 & 3,2 & 3,1 & 3,2 \\
20 & 3,1 & 3,1 & 3,1 & 3,1 & 3,0 & 3,0 & 3,0 & 3,1 & 3,1 & 3,1 & 3,0 & 3,1 \\
21 & 3,0 & 3,0 & 3,0 & 2,8 & 2,9 & 2,9 & 2,9 & 3,0 & 3,0 & 2,9 & 2,9 & 3,0 \\
22 & 2,9 & 2,9 & 2,9 & 2,7 & 2,8 & 2,8 & 2,8 & 2,9 & 2,9 & 2,8 & 2,9 & 2,9 \\
23 & 2,9 & 2,9 & 2,8 & 2,7 & 2,8 & 2,8 & 2,8 & 2,9 & 2,8 & 2,8 & 2,8 & 2,8 \\
\hline
\end{tabular}

\section{Strato F1}

Come accennato poco fa, lo strato F1 è ben riconoscibile a Roma soltanto nelle ore diurne dei mesi da Aprile ad Agosto. Nel periodo preso in esame la frequenza critica ordinaria massima, occorrente intorno al mezzogiorno solare vero, ha variato tra circa 4 e circa $7 \mathrm{MHz}$ (fig. 4), cui corrispondono densità elettroniche massime di circa $2 \cdot 10^{11}$ e circa $6 \cdot 10^{11} \mathrm{~m}^{-3}$.

Lo strato $\mathrm{F} 1$ è, come si sa, uno strato a controllo solare piuttosto regolare, il cui andamento segue abbastanza bene le modalità previste nella nota teoria di Chapman sulla fotoionizzazione ionosferica; non potendo fare medie mobili annue, ci siamo limitati a verificare se gli andamenti della frequenza critica ordinaria, foF1, sono in accordo con la teoria anzidetta. I risultati, sostanzialmente positivi, delle verifiche statistiche sono riportati nella fig. 4, per quanto riguarda la dipendenza di foF1 alle ore 12 , foF $1_{12}$, da $R$, e nella fig. 7 , per quanto riguarda gli andamenti diurni, mese per mese, di foF2 per $R=0$ e $R=100$; per 
verificare la dipendenza di foF1 dalla distanza zenitale $\chi$ del Sole, che secondo la teoria di Chapman dovrebbe essere di proporzionalità a $(\cos \chi)^{0,25}$, ̀̀ stato costruito un correlogramma che ha portato alla seguente relazione:

$$
\log _{10} \text { foF } 1=0,645+8,0 \cdot 10^{-4} R+0,20 \log _{10} \cos \chi,
$$

foF1 essendo in MHz. Nella Tab. III sono riportati i valori orari di $\log _{10} \cos \chi$ a Roma, il 15 di ogni mese. La formula derivante dalla teoria di Chapman è un po' diversa, non solo, come poco fa ricordato, per il diverso valore dell'esponente di $\cos \chi$, ma anche per la dipendenza da $R$. La [7], peraltro, ̀̀ di maneggio molto comorlo, il che è un fatto apprezzabile quando essa venga assunta, in luogo delle curve della fig. 7, per definire l'andamento normale della caratteristica ionosferica foF1 sulla verticale di Roma.

Per quanto riguarda l'altezza virtuale minima, l'andamento medio di essa è rappresentato, come accennato precedentemente, dalla curva $c$ della fig. 6 .

Tabella III - Parte decimale dei valori diuni di $\log _{10} \cos \%$ a Roma, IL 15 DI OGNI MESE; LA PARTE INTERA ì --0, SALVO PER I VALORI SOTTOLINEATI, CUI COMPETE I.A PARTE INTERA --I

\begin{tabular}{|c|c|c|c|c|c|c|c|c|c|c|c|c|}
\hline & Gen & Feb & Mar & Apr & Mag & Giu & Lug & Ago & Set & Ott & Nov & Dic \\
\hline 05 & & & & & 886 & 244 & 678 & & & & & \\
\hline 06 & & & & 113 & 710 & 633 & 703 & 924 & 699 & & & \\
\hline 07 & & & 983 & 572 & 421 & 386 & 421 & 511 & 672 & 971 & & \\
\hline 08 & 276 & 838 & 539 & 348 & 260 & 238 & 260 & 314 & 404 & 548 & 801 & 174 \\
\hline 09 & 686 & 514 & 345 & 217 & 195 & 140 & 154 & 193 & 259 & 362 & 520 & 676 \\
\hline 10 & 484 & 359 & 234 & 137 & 089 & 076 & 086 & 116 & 172 & 260 & 386 & 491 \\
\hline 11 & 386 & 278 & 173 & 091 & 051 & 039 & 045 & 072 & 124 & 208 & 321 & 403 \\
\hline 12 & 348 & 243 & 147 & 074 & 037 & 024 & 028 & 053 & 107 & 193 & 304 & 376 \\
\hline 13 & 356 & 246 & 153 & 081 & 046 & 031 & 034 & 060 & 119 & 213 & 330 & 396 \\
\hline 14 & 413 & 290 & 190 & 118 & 079 & 060 & 062 & 091 & 161 & 271 & 404 & 472 \\
\hline 15 & 541 & 382 & 268 & 186 & 134 & 115 & 115 & 151 & 240 & 381 & $\mathbf{5 5 6}$ & 634 \\
\hline 16 & 812 & 551 & 401 & 297 & 234 & 200 & 199 & 249 & 372 & 583 & 889 & 036 \\
\hline 17 & & 955 & 650 & 479 & 382 & 329 & 327 & 404 & 607 & 086 & & \\
\hline 18 & & & 456 & 845 & 633 & 533 & 532 & 680 & 252 & & & \\
\hline 19 & & & & & 301 & 939 & 947 & 658 & & & & \\
\hline
\end{tabular}


Come si sa, la regione $\mathrm{E}$ normale segue piuttosto bene gli andamenti a breve e a lungo termine previsti dalla teoria di Chapman; trattandosi di una regione diurna permanente nel corso dell'anno, è stato possibile fare per essa un'analisi statistica più dettagliata di quella fatta per i dati F1.

$\mathrm{Nel}$ periodo considerato la frequenza critica ordinaria massima, occorrente intorno al mezzogiorno solare vero, ha variato all'incirca tra 2,6 e 4,5 MHz, cui corrispondono densità elettroniche massime di circa $8,4 \cdot 10^{10}$ e circa $2,5 \cdot 10^{11} \mathrm{~m}^{-3}$.

Nelle già citate figg. 4 e 7 sono riportati gli andamenti della frequenza critica ordinaria, foE, e precisamente nella prima l'andamento della media mobile annua alle ore 12 , fo $\mathrm{E}_{12}$, con $R$ e nella seconda gli andamenti diurni mensili per $R=0$ e per $R=100$. Come fatto per la caratteristica ionosferica foF1, dal correlogramma tra foE e $\cos \chi$ si è ricavata la seguente relazione:

$$
\log _{10} \text { foE }=0,52+5,8 \cdot 10^{-4} R+0,29 \log _{10} \cos \chi
$$

foE essendo in MHz. Questa relazione, per la quale valgono le osservazioni fatte a proposito della [7], può essere assunta, in luogo delle curve della fig. 7, per definire l'andamento normale della caratteristica ionosferica foE sulla verticale di Roma.

Per quanto riguarda l'altezza virtuale minima, h'E, la risoluzione delle letture normali degli ionogrammi dell'Osservatorio di RomaS. Alessio, che è di $\pm 5 \mathrm{~km}$ in altezza virtuale, non consente di apprezzare altro che le variazioni ai crepuscoli, nelle ore meridiane la caratteristica $h^{\prime} \mathrm{E}$ presentandosi statisticamente costante al valore di $110 \mathrm{~km}$; ai crepuscoli, $\mathrm{h}^{\prime} \mathrm{E}$ è osservabile sino a valori di $140 \div 160 \mathrm{~km}$, ma l'interpretazione è resa difficile da frequenti stratificazioni, specie all'alba. 


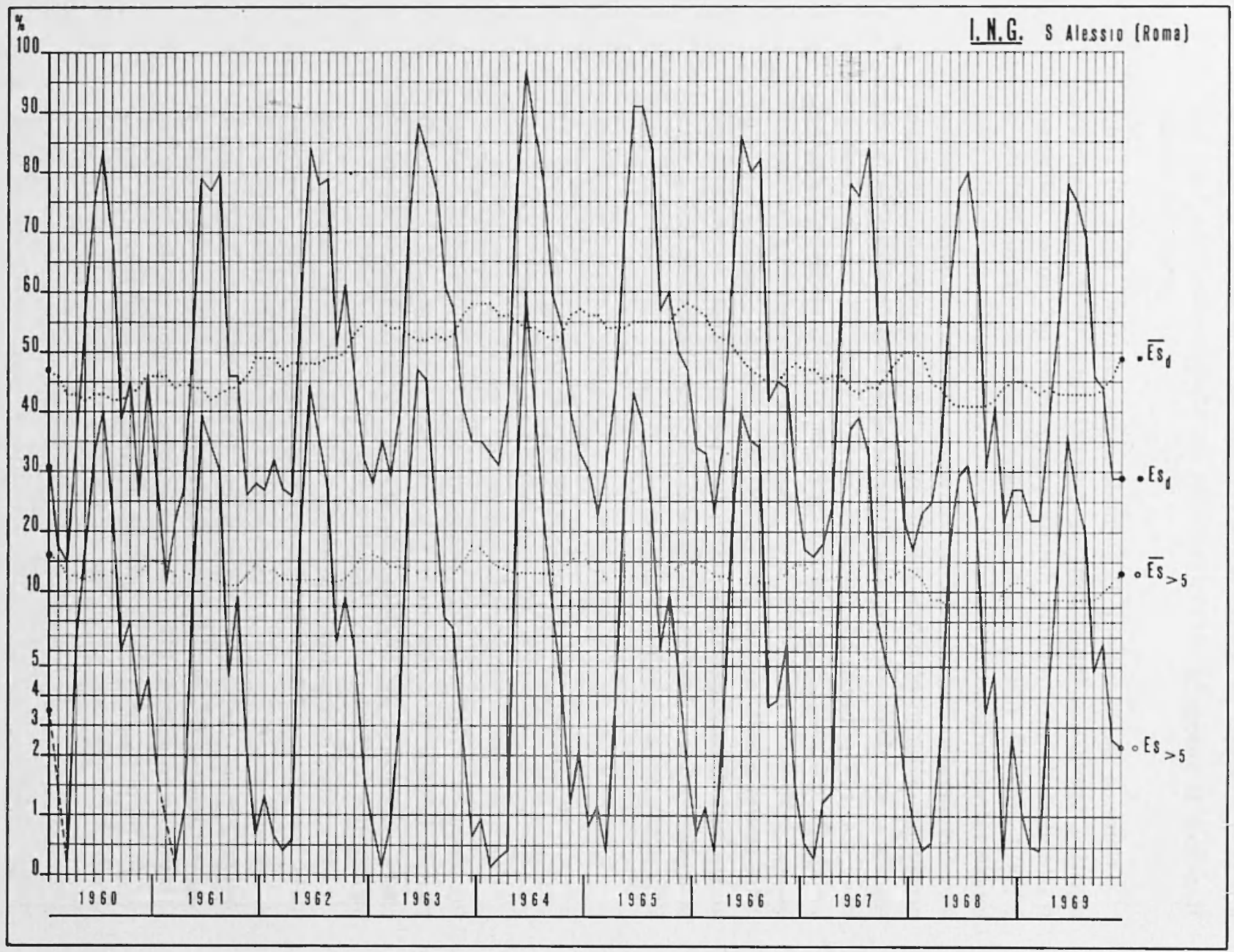

Fig. 8. - Andamento dei valori mensili e delle medie mobili annue (puntegrgiate) nel decennio 1960 -1969 dell'occorrenza temporale globale $\left(\mathrm{Es}_{d}\right)$ e con $f>5 \mathrm{MHz}\left(\mathrm{Es}_{>5}\right)$, dello strato $\mathrm{E}$ sporadico. 


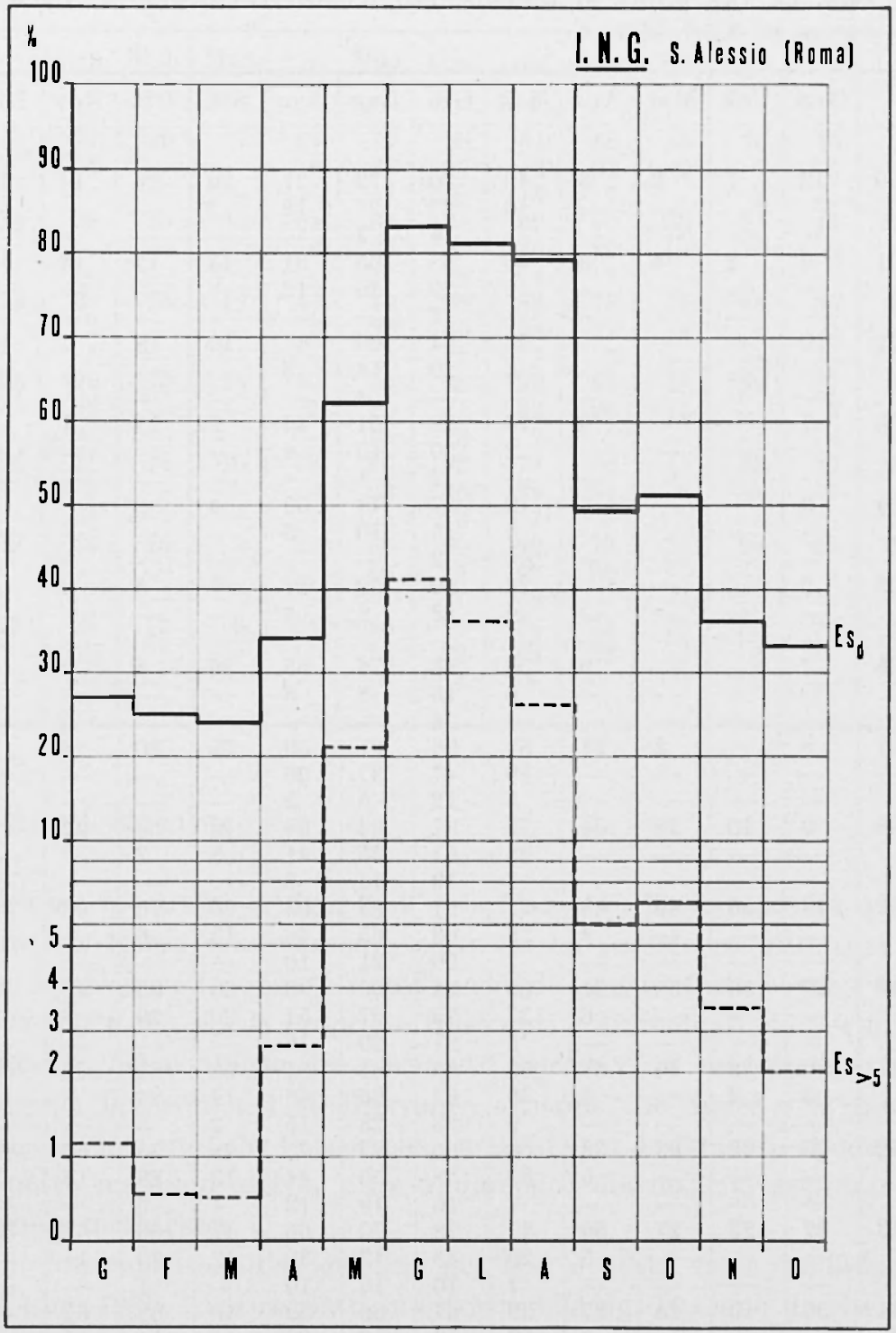

I'ig. 9. - Andamento annuo normale dell'occorrenza temporale, globale $\left(\mathrm{Es}_{d}\right)$ e con $f>5 \mathrm{MHz}\left(\mathrm{Es}_{>5}\right)$, dello strato $\mathrm{E}$ sporadico. 


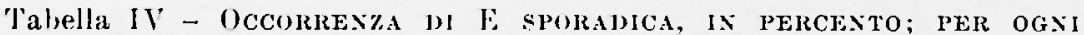
ORA, LE TRE RIGHE SI RIFERISCoNo, RISPETtivaMente, a fEs MaGGIOKE $113,5,7 \mathrm{MHz}$

\begin{tabular}{|c|c|c|c|c|c|c|c|c|c|c|c|c|}
\hline & Gen & Feb & Mar & Apr & Magr & Giu & Lugg & A goo & Set & ()tt & Nov & Dic: \\
\hline \multirow[t]{3}{*}{00} & 13 & 7 & 2 & 3 & 40) & 70 & 73 & 71 & 20 & 26 & 14 & 21 \\
\hline & - & - & - & - & 10 & 20 & 27 & 12 & 2 & - & - & - \\
\hline & $\overline{0}$ & $\overline{0}$ & - & 二 & $\overline{30}$ & (i) & 65 & $\bar{a}$ & $\overline{12}$ & $\overline{17}$ & $\overline{19}$ & $\overline{\bar{c}}$ \\
\hline \multirow[t]{3}{*}{01} & 9 & 2 & 4 & 3 & 36 & 68 & 65 & 61 & 13 & 17 & 13 & 16 \\
\hline & 一 & - & - & - & 7 & 20 & 20 & 12 & - & - & - & - \\
\hline & $\overline{10}$ & - & - & 一 & $\overline{0}$ & 2 & $\bar{c}$ & $\overline{15}$ & $\overline{10}$ & $\overline{10}$ & - & $\overline{1}$ \\
\hline \multirow[t]{2}{*}{02} & 10 & - & - & - & 25 & 64 & 60 & 47 & 10 & 18 & 7 & 10 \\
\hline & 二 & - & - & - & 6 & 20 & 14 & 5 & - & - & - & - \\
\hline \multirow[t]{3}{*}{03} & 7 & - & - & $\overline{2}$ & $\overline{24}$ & 58 & $5 i$ & $\overline{45}$ & 9 & 18 & 7 & 3 \\
\hline & 一 & - & - & - & 2 & 20 & 15 & 4 & - & - & - & - \\
\hline & - & - & - & - & $\overline{-}$ & 2 & 1 & $\bar{\sigma}$ & - & 一 & 一 & - \\
\hline \multirow[t]{3}{*}{04} & 3 & - & 一 & 4 & 19 & 57 & 48 & 32 & 4 & 9 & - & 2 \\
\hline & - & - & - & - & 5 & 15 & 10 & 5 & - & - & - & - \\
\hline & 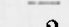 & - & - & - & $\bar{a}$ & 2 & $\overline{50}$ & $\vec{a}$ & -- & - & 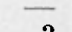 & T \\
\hline \multirow[t]{2}{*}{05} & 2 & 一 & - & - & 26 & 60 & 50 & 31 & 7 & 5 & 3 & 4 \\
\hline & 二 & 二 & - & - & 4 & 13 & 8 & 4 & 二 & - & - & - \\
\hline \multirow[t]{3}{*}{06} & 5 & - & - & 9 & 53 & 83 & 76 & 55 & 15 & 4 & 5 & 5 \\
\hline & 一 & - & - & - & 6 & 16 & 8 & 6 & - & - & - & - \\
\hline & - & 一 & - & - & - & 4 & - & - & - & - & $\longrightarrow$ & - \\
\hline \multirow[t]{3}{*}{07} & 8 & - & 2 & 21 & 81 & 96 & 91 & 89 & 59 & 20 & 6 & 7 \\
\hline & 一 & 一 & - & - & 19 & 47 & 37 & & - & - & - & - \\
\hline & - & - & - & - & 5 & 12 & 5 & & - & - & - & - \\
\hline \multirow[t]{3}{*}{08} & 9 & 10 & 28 & 51 & 77 & 97 & 94 & & 78 & 58 & 22 & 12 \\
\hline & - & - & - & 一 & 25 & 61 & 5 & 4 & 8 & 2 & - & 一 \\
\hline & - & - & - & - & 7 & & & & - & - & - & - \\
\hline \multirow[t]{3}{*}{09} & 19 & 36 & 49 & 48 & 71 & 91 & 93 & & 65 & 76 & 66 & 30 \\
\hline & - & - & - & 4 & 36 & 62 & 65 & & 13 & 6 & - & 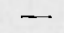 \\
\hline & $\overline{0}$ & - & - & - & 7 & & & & - & - & $\overline{-}$ & $\bar{\sim}$ \\
\hline \multirow[t]{3}{*}{10} & 27 & 56 & 50 & 31 & 69 & 91 & 90 & & 60 & 85 & 72 & 35 \\
\hline & & & - & 6 & 37 & 73 & 67 & & 20 & 20 & 3 & 2 \\
\hline & $\overline{0}$ & $\overline{-}$ & $\overline{0}$ & $\overline{0}$ & $\overline{\mathbf{5}}$ & & & & & - & $\overline{\sigma 0}$ & $\overline{0}$ \\
\hline \multirow[t]{3}{*}{11} & 36 & 36 & 36 & 37 & 62 & 85 & 88 & & 58 & 81 & 72 & 36 \\
\hline & 3 & 2 & & 4 & 36 & 67 & 65 & & 14 & 24 & 8 & - \\
\hline & $\overline{0}$ & $\bar{n}$ & & $\overline{0}$ & 9 & & & & 2 & 5 & $\overline{-}$ & $\overline{1}$ \\
\hline \multirow[t]{3}{*}{12} & 29 & 29 & 33 & 33 & 56 & 85 & 8 & & 48 & 70 & 56 & 45 \\
\hline & - & 一 & - & 6 & 35 & 5 & & & 13 & 28 & 15 & 2 \\
\hline & 二 & 二 & - & $\overline{-}$ & 7 & 1 & & & 4 & 9 & $\bar{F}$ & ․ \\
\hline \multirow[t]{3}{*}{13} & 27 & 23 & 27 & 30 & 47 & 78 & 70 & & 47 & 66 & 52 & 38 \\
\hline & & 一 & - & 5 & 29 & 45 & 4 & & 12 & 20 & 11 & - \\
\hline & & - & - & - & 7 & 10 & & & 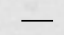 & 4 & - & - \\
\hline \multirow[t]{3}{*}{14} & 30) & 19 & 25 & 27 & 39 & 67 & 60 & & 40 & 68 & 67 & 40 \\
\hline & & & - & 2 & 23 & 4 & & 2 & 11 & 14 & 7 & - \\
\hline & & - & - & - & 8 & 15 & 12 & & 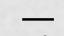 & 4 & 一 & 一 \\
\hline \multirow[t]{3}{*}{15} & 26 & 28 & 35 & 30) & 52 & 77 & 7 & & 49 & 66 & 41 & 35 \\
\hline & & & - & 4 & 26 & 41 & 3 & 2 & 7 & 8 & 4 & - \\
\hline & - & - & - & & 5 & 17 & 10 & $\theta$ & - & 3 & - & - \\
\hline \multirow[t]{3}{*}{16} & 28 & 27 & 42 & 45 & 70 & 79 & 76 & 77 & 63 & 66 & 40 & 25 \\
\hline & 2 & & - & 4 & 35 & 48 & 38 & 27 & 4 & 7 & - & - \\
\hline & & & - & - & 6 & 18 & 12 & 4 & 一 & - & - & - \\
\hline
\end{tabular}


Segue Tabella IV

\begin{tabular}{|c|c|c|c|c|c|c|c|c|c|c|c|c|}
\hline & Gen & Feb & Mar & $A_{p}$ & Mag & Giu & Lug & Ago & Set & Ott & Nov & Dic \\
\hline \multirow[t]{3}{*}{17} & 21 & 30 & 37 & 67 & 79 & 86 & 84 & 86 & 58 & 70 & 40 & 29 \\
\hline & 2 & - & - & 6 & 37 & 47 & 46 & 32 & 2 & 7 & 5 & 2 \\
\hline & - & - & - & - & 11 & 21 & 13 & 7 & - & 2 & - & - \\
\hline \multirow[t]{3}{*}{18} & 28 & 26 & 33 & 58 & 87 & 95 & 89 & 90 & 58 & 54 & 47 & 27 \\
\hline & 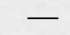 & - & - & 6 & 33 & 51 & 40 & 29 & 4 & 10 & 3 & 7 \\
\hline & - & - & - & - & 11 & 20 & 14 & 7 & - & - & - & - \\
\hline \multirow[t]{3}{*}{19} & 31 & 24 & 19 & 41 & 76 & 90 & 88 & 78 & 51 & 48 & 30 & 38 \\
\hline & 3 & - & - & 2 & 27 & 40 & 33 & 27 & 4 & 6 & 3 & 5 \\
\hline & - & $\overline{-}$ & - & 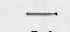 & 10 & 11 & 7 & $\mathbf{5}$ & $\overline{-}$ & - & - & - \\
\hline \multirow[t]{3}{*}{20} & 29 & 16 & 15 & 30 & 64 & 82 & 83 & 83 & 48 & 39 & 31 & 37 \\
\hline & 2 & - & $\ldots$ & 2 & 20 & 41 & 32 & 26 & 13 & -... & 3 & 7 \\
\hline & - & - & - & - & 5 & 18 & 7 & 7 & - & $\cdots$ & - & - \\
\hline \multirow[t]{3}{*}{21} & 31 & 14 & 10 & 19 & 60 & 83 & 79 & 76 & 45 & 33 & 29 & 35 \\
\hline & 1 & - & - & 4 & 23 & 46 & 38 & 34 & 7 & - & - & 4 \\
\hline & - & - & - & - & 5 & 10 & 7 & 6 & - & - & - & - \\
\hline \multirow[t]{3}{*}{22} & 28 & 15 & 9 & 21 & 55 & 80 & 80 & 79 & 35 & 28 & 23 & 37 \\
\hline & $\rightarrow$ & 一 & 一 & - & 19 & 44 & 29 & 30 & 4 & - & - & 2 \\
\hline & - & - & - & - & - & 7 & 6 & - & - & - & - & - \\
\hline \multirow[t]{3}{*}{23} & 26 & 12 & 6 & 14 & 55 & 79 & 78 & 74 & 29 & 30 & 20 & 29 \\
\hline & - & - & - & - & 15 & 29 & 35 & 22 & 3 & 2 & - & - \\
\hline & & - & 一 & - & - & 4 & 4 & - & - & - & - & - \\
\hline
\end{tabular}

\section{STRATO E SPORADICO}

Come mostrano le figg. 8, 9 e la Tab. IV, l'occorrenza di questo strato è a Roma relativamente alta; ciò ha particolare rilevanza sul piano applicativo in quanto significa che di questo strato occorre tener debito conto, sia per le proprietà riflettenti e difiondenti sia per quelle occultanti, nel computo dei parametri operativi di radiocollegamenti ionosferici nell'area del Mediterraneo centrale. Le figure e la tabella anzidette mostrano che l'occorrenza presenta un andamento stagionale, con netto massimo estivo, e un andamento diurno, con apprezzabile massimo meridiano.

Relativamente all'occorrenza dei tipi è stata fatta un'indagine per l'anno 1970, i cui risultati, mostrati nella tig. 10, sono da ritenersi generalmente validi per la zona di Roma. Come si verle, il tipo "piatto" (f notturno, 1 diurno) è praticamente il solo presente di notte, mentre di giorno nei mesi estivi cede il posto ai tipi c e h, che si presentano dunque come tipi a vago controllo solare. Il tipo "cuspidato" c, caratterizzato, come si sa, da una cuspide allo stacco dalla regione $\mathrm{E}$ normale, ha un andamento diurno con un massimo antimeridiano, 


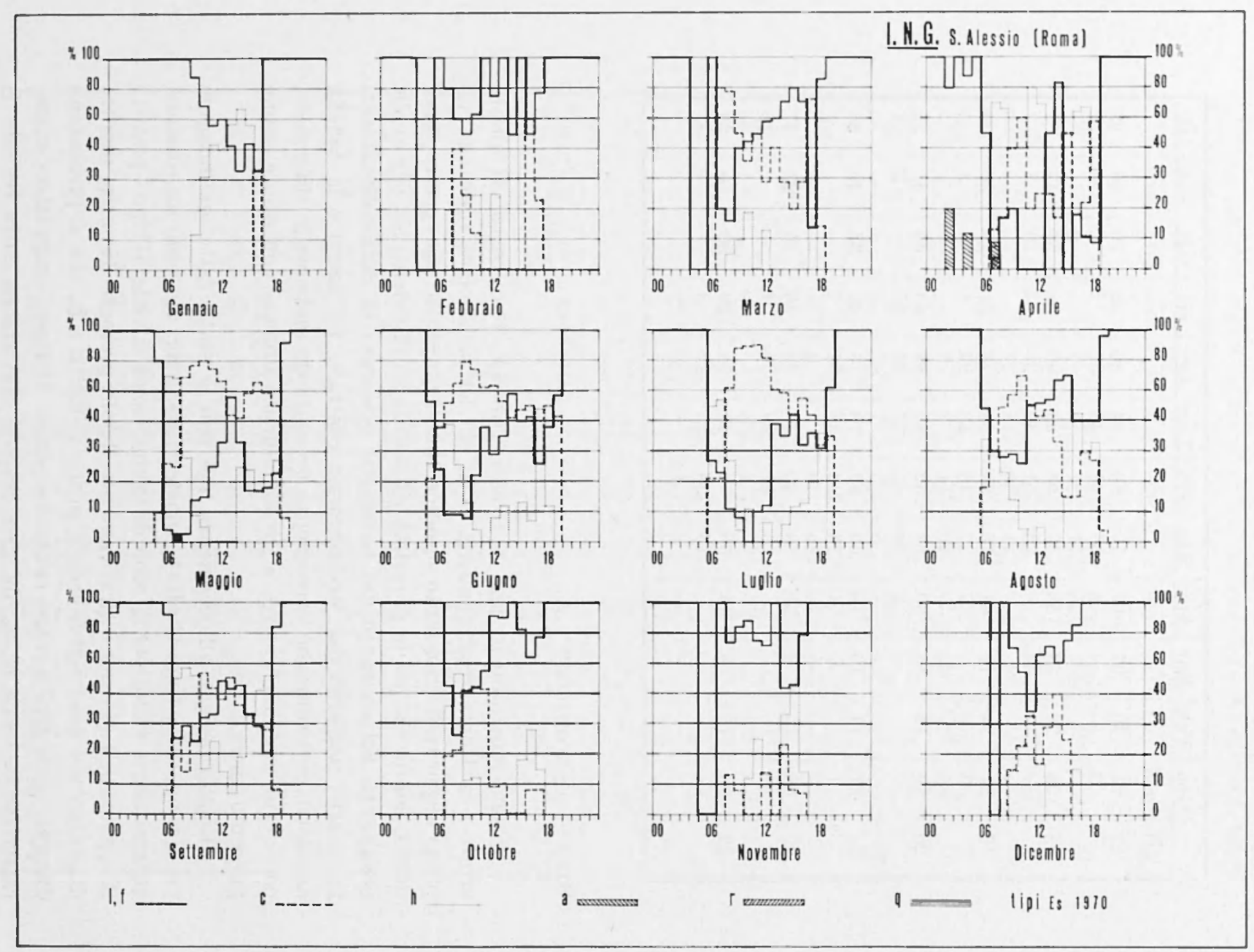

Fig. 10. - Andamento dell'occorrenza relativa dei tipi di E sporadica nel 1970. 
laddove il tipo "alto " h, caratterizzato da un sensibile divario di quota dalla regione $\mathrm{E}$ normale, sembra mostrare una depressione meridiana. Praticamente inesistenti i tipi aurorale (a), equatoriale (q), ritardante (r).

Per quanto riguarda l'altezza virtuale, questa s'è mantenuta sul valore medio di $130 \mathrm{~km}$ per i tipi più frequenti.

\section{REGIONE I)}

Poiché la frequenza critica massima di questa regione è sempre nettamente inferiore alla frequenza minima di lavoro delle sonde usate all'Osservatorio di Roma-S. Alessio, le uniche informazioni ottenibili dagli ionogrammi sono quelle ricavabili dalla caratteristica ionosferica fmin, cioè dalla frequenza minima a cui cominciano ad apparire echi sullo ionogramma. Ancorché tale caratteristica abbia notevoli incertezze intrinseche, fra l'altro perché dipende anche da caratteristiche strumentali (potenza irradiata e sensibilità in ricezione), i dati disponibili si sono rivelati atti a una soddisfacente verifica del presunto andamento "alla Chapman " di tale regione; precisamente, da correlogrammi fmin-cos $\chi$ è stato dedotto il valore medio 0,23 per l'esponente di $\cos \chi$, contro il valore di 0,25 voluto dalla teoria di Chapman, nella legge di dipendenza del tasso di fotoionizzazione della regione dalla distanza zenitale $\chi$ del Sole.

Nulla invece si può dire riguardo all'altezza della regione.

\section{BIBLIOGRAFIA}

(1) Bolle A., 1940. - Altezze di riflessione delle radioonde a Roma dal (iennaio al Settembre 1939, "P'ubblic. dell'ING ", n. 26.

(2) Bolle A., Silleni \$., Trberio (Ł. A., 1949. - Registrazioni ionosferiche dal settembre 1948 al marzo 1949, "Annali di Geofisica ", II, 3.

(3) Dominici P., 1954. - Andamento della ionosfera a Roma nel periodo 1948 . 19.5, "Annali di Geofisica ", VII, 4.

(4) Domisici P., 1955. - Talori mediani mensili delle caratteristiche ionosje. riche nel periodo marzo 19 49 -aprile 19.53, "Annali di Geofisica. ", VIII, 3.

$\left.{ }^{5}{ }^{5}\right) \mathrm{R} A \mathrm{NZI}$ I., 1939. - Osservazioni ionosferiche eseguite a Roma dallagosto al novembre 19.38, "Pubblic. dell'ING ", n. 8.

${ }^{6}$ ) Ranzi I., 1940. - Frequenze critiche ionosferiche osservale a Roma dal dicembre 1935 al settembre 1939, "Pubblic. dell'ING ", n. 25. 